\title{
Fatores de risco relacionados à pneumonia associada a ventilação mecânica: revisão da literatura
}

\author{
Risk factors related to pneumonia associated with mechanical ventilation: literature review \\ Factores de riesgo relacionados con la neumonía asociada a la ventilación mecánica: \\ revisión de la literatura
}

Maria Teperino Pinho ${ }^{1 *}$, Caroline Macedo Calegario dos Santos ${ }^{1}$, Bruna Cavalcante Servio ${ }^{1}$, Lídia Miranda Brinati ${ }^{2}$, Luana Vieira Toledo ${ }^{1}$, Patrícia de Oliveira Salgado'.

\section{RESUMO}

Objetivo: Identificar na literatura fatores de risco relacionados à Pneumonia Associada à Ventilação Mecânica (PAVM) em pacientes adultos. Métodos: Revisão da literatura cuja busca ocorreu nas bases de dados Literatura Latino Americana e do caribe em Ciências da Saúde, U.S. National Library of Medicine e Cumulative Index to Nursing and Allied Health Literature. Os critérios de inclusão foram artigos publicados entre os anos de 2005 a 2019, nas línguas portuguesa, inglesa e espanhola, que abordavam os riscos para a PAVM em pacientes adultos críticos. Foram excluídos os artigos que abordavam sobre terapias medicamentosas, microrganismos, atualizações sobre o tema, consensos de opiniões e bundles sobre PAVM. Resultados: Os principais fatores de risco associados ao desenvolvimento da PAVM são doenças respiratórias prévias, traumas, idade, uso prévio e inadequado de antibióticos, exposição ao ventilador mecânico, tempo prolongado de uso da VM, ausência de higiene oral do paciente, falta de higiene das mãos do profissional e o incorreto posicionamento no leito. Considerações finais: A equipe multiprofissional tem uma responsabilidade considerável no controle e prevenção da PAVM, assim, é necessário que todos os profissionais conheçam os fatores de risco associados e prestem assistência buscando prevenir o desenvolvimento desse problema.

Palavras-chave: Pneumonia associada à ventilação mecânica, Fatores de risco, Unidades de terapia intensiva.

\section{ABSTRACT}

Objective: To identify in the literature risk factors related to Pneumonia Associated with Mechanical Ventilation (VAP) in adult patients. Methods: Literature review that searched the Latin American and Caribbean Health Sciences, U.S. National Library of Medicine and Cumulative Index to Nursing and Allied Health Literature databases. The inclusion criteria were articles published between the years 2005 to 2019, in Portuguese, English and Spanish, which addressed the risks for VAP in critically ill adult patients. Articles that addressed drug therapies, microorganisms, updates on the topic, consensus of opinions and bundles on VAP were excluded. Results: The main risk factors associated with the development of VAP are previous respiratory diseases, trauma, age, previous and inappropriate use of antibiotics, exposure to a mechanical ventilator, prolonged use of MV, lack of oral hygiene for the patient, poor hygiene the hands of the professional and the incorrect positioning on the bed. Final considerations: The multiprofessional team has a considerable responsibility in the control and prevention of VAP, thus, it is necessary that all professionals know the associated risk factors and provide assistance seeking to prevent the development of this problem.

Keywords: Pneumonia ventilador-associated, Risk factors, Intensive care units.

\section{RESUMEN}

Objetivo: Identificar en la literatura los factores de riesgo relacionados con la neumonía asociada a la ventilación mecánica (NAV) en pacientes adultos. Métodos: Revisión de la literatura que buscó en las bases de datos de Ciencias de la Salud de América Latina y el Caribe, la Biblioteca Nacional de Medicina de EE. Los criterios de inclusión fueron artículos publicados entre los años 2005 a 2019, en portugués, inglés y español, que abordaban los riesgos de NAV en pacientes adultos críticamente enfermos. Se excluyeron los artículos que abordaban terapias farmacológicas, microorganismos, actualizaciones sobre el tema, consenso de opiniones y paquetes sobre VAP. Resultados: Los principales factores de riesgo asociados al desarrollo de NAV son enfermedades respiratorias previas, trauma, edad, uso previo e inadecuado de antibióticos, exposición a ventilador mecánico, uso prolongado de VM, falta de higiene bucal del paciente, mala higiene del manos del profesional y el posicionamiento incorrecto en la cama. Consideraciones finales: El equipo multiprofesional tiene una responsabilidad considerable en el control y prevención de la NAV, por lo que es necesario que todos los profesionales conozcan los factores de riesgo asociados y brinden asistencia buscando prevenir el desarrollo de este problema.

Palabras clave: Neumonía asociada al ventilador, Factores de riesgo, Unidades de cuidados intensivos.

${ }^{1}$ Universidade Federal de Viçosa (UFV), Viçosa - MG. *E-mail: maria.teperinopinho@gmail.com

2 Hospital São Sebastião, Viçosa - MG. 


\section{INTRODUÇÃO}

Pacientes hospitalizados em Unidades de Terapia Intensiva (UTIs) são expostos, constantemente, a procedimentos e seus riscos, mediante a complexidade da unidade. As Infecções Relacionadas à Assistência à Saúde (IRAS) constituem o evento adverso mais comum no cuidado em saúde em todo mundo, afetando $7 \%$ dos pacientes em países desenvolvidos e $10 \%$ nos países em desenvolvimento. A infecção das vias urinárias, do sítio cirúrgico, da corrente sanguínea e a pneumonia os tipos mais frequentes (DUTRA LA, et al., 2019).

A Pneumonia Associada à Ventilação Mecânica (PAVM) é a infecção pulmonar que ocorre de 48 a 72 horas após exposição a intubação endotraqueal e instituição da ventilação mecânica. Tem como agente causador, um microrganismo que não estava presente ou incubado e, sua ocorrência, geralmente, decorre da aspiração de secreções das vias áreas superiores, do refluxo gastrintestinal e da inoculação de material exógeno contaminado (SILVA RM, et al., 2011; MOTA EC, et al., 2017). Caracteriza-se por inflamação aguda ou crônica do parênquima pulmonar, podendo ser de origem bacteriana, fúngica, viral ou de outros processos que podem acometer o aparelho respiratório (MATOSO LML e CASTRO CHA, 2013).

Em especial, a pneumonia tem seu risco aumentado diretamente nos pacientes em uso da Ventilação Mecânica (VM) (LEAL GA, et al., 2017). A PAVM é considerada a segunda infecção mais frequente nas UTIs americanas e a mais frequente na Europa. A notificação da ocorrência da PAVM nas UTI's brasileiras tornouse obrigatória somente a partir do ano de 2017 e um estudo nacional demonstra que essa é uma das mais incidentes infecções hospitalares, resultando em altos índices de internações prolongadas (NASCIMENTO TBP, et al., 2017). Dados do Estado de São Paulo, em 2015, indicam que a incidência da PAVM, foi de 9,87 casos por 1.000 dias de uso de ventilador em UTI adulto. Para UTIs de hospital de ensino, com 13,40 casos por 1.000 ventilador/dia e nas UTIs de hospitais privados 6,56 casos de PAVM foram diagnosticados, sendo que $41,17 \%$ dos pacientes da UTI estavam em uso da ventilação mecânica (ANVISA, 2017).

Muitos fatores estão associados ao risco de desenvolvimento de PAVM, sendo estes modificáveis ou não. Os fatores de risco modificáveis estão relacionados à microbiota da própria UTI, justificando a importância de conhecer os agentes patogênicos mais frequentes em cada unidade específica. Entre os não modificáveis, observa-se a idade, escore de gravidade avaliado após a admissão do paciente e presença de comorbidades prévias (SANTOS CR, et al., 2018). Apesar da discrição de alguns fatores associados, a compreensão do agravo ainda é deficiente, uma vez que a coleta de dados relacionada a esse problema em UTIs no Brasil é pouco precisa, com ausência de padronização dos dados (ANVISA, 2017).

Nota-se que a enfermagem assumiu, desde Florence Nightingale, a importante função na prevenção e controla das IRAS e no desenvolvimento de práticas de apoio epidemiológicos. Diante disso, é importante destacar que a prática de enfermagem precisa ser baseada em evidências científicas e sobretudo deve buscar a redução da incidência de complicações e agravos, como a pneumonia associada à ventilação mecânica (BATISTA JF, et al., 2013)

Nessa perspectiva, compreendendo a necessidade de padronização das condutas adotadas nas UTI's e visando a promoção da assistência de enfermagem de qualidade, buscou-se a resposta para a seguinte indagação: quais os fatores de risco para o desenvolvimento da Pneumonia Associada à Ventilação Mecânica apresentados na literatura? Sendo assim, este trabalho tem por objetivo identificar na literatura quais são os fatores de risco relacionados à PAVM em pacientes adultos críticos.

\section{MÉTODOS}

Trata-se de uma revisão da literatura integrativa realizada seguindo as seguintes etapas: identificação do tema e seleção da questão/hipótese de pesquisa; seleção dos critérios para inclusão/exclusão dos estudas na busca literária; definição das informações a serem observadas nos estudos; avaliação dos estudos que foram incluídos na revisão; análise e interpretação dos resultados (MENDES KDS, et al., 2008).

O levantamento das publicações indexadas foi realizado no período julho a agosto de 2019 nas bases bibliográficas digitais, através do acesso da Biblioteca Virtual da Saúde (BVS) por meio de publicações encontradas na Literatura Latino Americana e do Caribe em Ciências da Saúde (LILACS), U.S. National Library of Medicine (PUBMED) e Cumulative Index to Nursing and Allied Health Literature (CINAHL). Para as 
pesquisas foram utilizados, em inglês, os seguintes descritores: "Ventilator-associated pneumonia", "Nosocomial infection" AND "Risk factors"; em português "Pneumonia associada ao ventilador", "Infecção hospitalar" e "Fatores de risco" e em espanhol, "Neumonía asociada al ventilador "," Infección nosocomial " Y " Factores de riesgo ".

Os critérios de inclusão foram artigos publicados entre os anos de 2005 a 2019, nas línguas portuguesa, inglesa e espanhola e que trouxessem na abordagem os fatores de risco para a Pneumonia associada a ventilação mecânica em pacientes adultos críticos. Destaca-se que o critério artigo publicado entre 2005 e 2019 foi estabelecido uma vez que se pretende com esse trabalhoidentificar os trabalhos mais recentes sobre o assunto. Os critérios de exclusão foram artigos que descreviam terapias medicamentosas, que estudavam os microrganismos envolvidos na patologia em questão e atualizações, consensos de opiniões, bundles sobre o tema e estudos do tipo revisão de literatura.

A avaliação da qualidade metodológica dos estudos foi realizada com base nos itens das iniciativas Strobe (Strengthening the Reporting of Observational Studies in Epidemiology), Consort (Consolidated Standards of Reporting Trials) ou Prisma (Preferred Reporting Items for Systematic Reviews and Meta-Analyses), de acordo com o delineamento do estudo. Considerou-se ter adequada qualidade metodológica os artigos que apresentaram concordância com $50 \%$ ou mais dos itens da iniciativa utilizada. Essa avaliação foi realizada por duas avaliadoras, de forma independente, e as inconsistências foram resolvidas por uma terceira avaliadora independente (Figura 1).

Figura 1 - Fluxograma de seleção dos artigos.

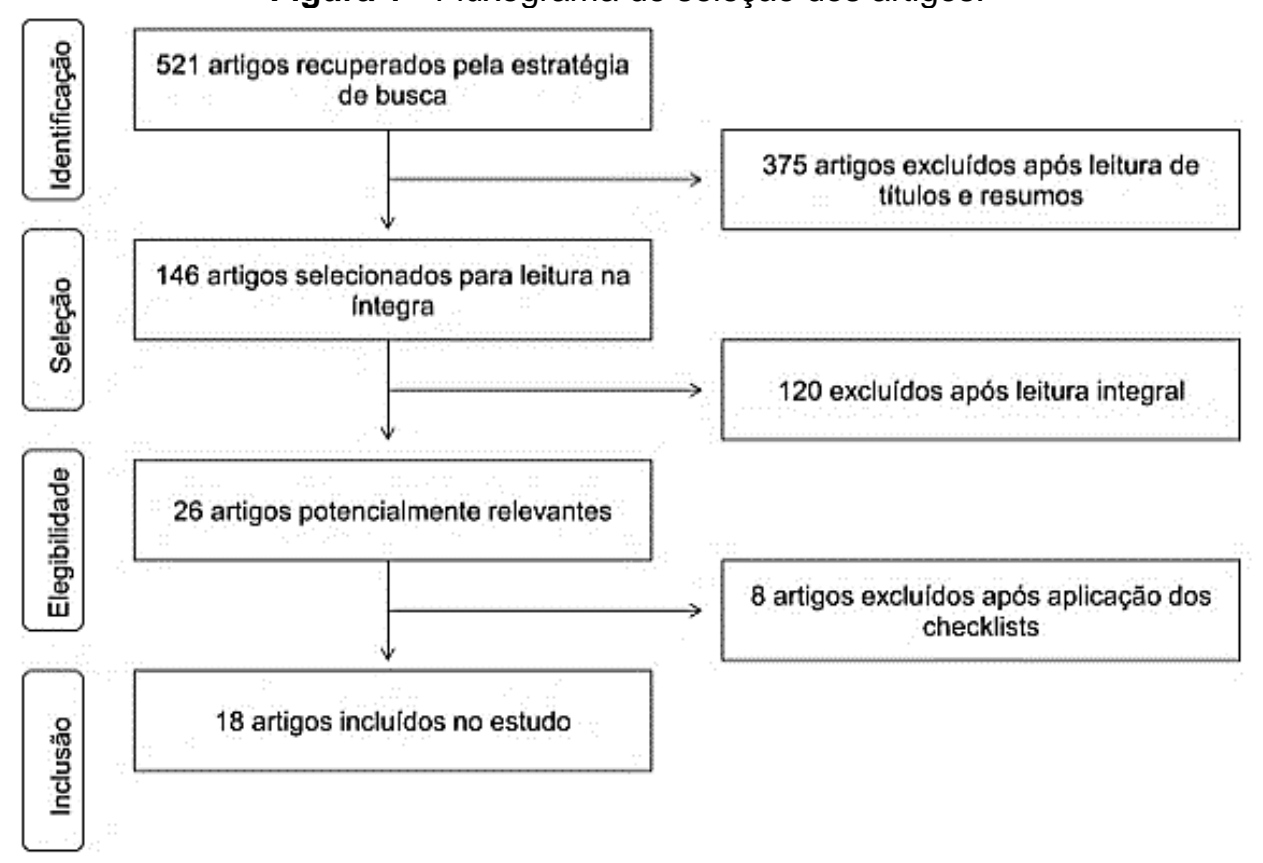

Fonte: Pinho MT, et al., 2021.

Para a coleta dos dados utilizou-se um instrumento elaborado pelas pesquisadoras contendo: título do artigo, título do periódico, autores, país em que o estudo foi realizado, idioma, o ano de publicação e os descritores. Além disso, o instrumento continha informações metodológicas, como os objetivos do estudo, a fonte/bases de dados, o tipo de estudo e de publicação, o delineamento, amostra, duração do estudo, resultados, conclusão, recomendações e limitações da pesquisa. Os dados foram extraídos por três avaliadoras de forma independente. As inconsistências foram resolvidas por uma quarta avaliadora.

\section{RESULTADOS}

Dezoito estudos preencheram os critérios de elegibilidade e permaneceram na análise. Os estudos foram publicados na língua portuguesa (8- 44,44\%), inglesa (8- 44,44\%) e espanhola (2- 11,11\%). Os países de origem foram: Brasil (9-50\%), Estados Unidos (1-5,55\%), Alemanha (1-5,55\%), Coréia do Sul (1-5,55\%), Turquia (2-11,11\%), França (2-11,11\%) e Colômbia (2-11,11\%) (Quadro 1). 


\begin{tabular}{|c|c|c|}
\hline Título do Artigo, País e Ano de Publicação & Objetivo & Fatores de risco \\
\hline $\begin{array}{l}\text { Adesão às medidas de um bundle para } \\
\text { prevenção de PAVM Brasil, } 2014\end{array}$ & $\begin{array}{l}\text { Avaliar a adesão ao bundle de ventilação mecânica em } \\
\text { uma UTI e seu impacto nas taxas de PAVM. }\end{array}$ & $\begin{array}{l}\text { Intubação; Traqueostomia; Tempo de permanência na UTI; Posição da cabeceira; } \\
\text { Manejo do circuito do ventilador; Higiene oral; Pressão do balonete do tudo } \\
\text { orotraqueal. }\end{array}$ \\
\hline $\begin{array}{l}\text { Conhecimento dos profissionais de saúde na } \\
\text { UTI sobre prevenção de PAVM. Brasil, } 2010 \text {. }\end{array}$ & $\begin{array}{l}\text { Avaliar o conhecimento dos profissionais de saúde sobre } \\
\text { a prevenção da PAVM em duas UTI de dois hospitais } \\
\text { públicos de Fortaleza (CE). }\end{array}$ & $\begin{array}{l}\text { Idade avançada; Coma; Nível de consciência; Intubação e reintubação traqueal; } \\
\text { Imunodeficiência; Uso de drogas imunodepressoras; Choque; Gravidade da } \\
\text { doença; Antecedência de DPOC; Tempo prolongado de VM > } 7 \text { dias; Aspirado do } \\
\text { condensado contaminado dos circuitos do ventilador; Desnutrição; Contaminação } \\
\text { exógena; Antibioticoterapia como profilaxia; Colonização microbiana; Cirurgias } \\
\text { prolongadas; Aspiração de secreções contaminadas; Colonização gástrica e } \\
\text { aspiração desta; Ph gástrico > } 4 \text {. }\end{array}$ \\
\hline $\begin{array}{l}\text { Early- and late-onset ventilator-associated } \\
\text { pneumonia acquired in the intensive care unit: } \\
\text { comparison of risk factors. França, } 2008 .\end{array}$ & $\begin{array}{l}\text { Comparar fatores de risco para pneumonia associada à } \\
\text { ventilação mecânica precoce }(E) \text { e tardia }(L) \text {. }\end{array}$ & $\begin{array}{l}\text { Idade avançada; Antibioticoterapia previa; Hospitalização prévia; Infecção na } \\
\text { admissão. }\end{array}$ \\
\hline $\begin{array}{l}\text { Factores de riesgo para neumonia asociada al } \\
\text { ventilador en un hospital de tercer nivel de la } \\
\text { ciudad de Medellin: estudio de casos y } \\
\text { controles. Colômbia, 2008. }\end{array}$ & $\begin{array}{l}\text { Identificar os principais fatores de risco responsáveis pelo } \\
\text { desenvolvimento da PAV no Hospital Pablo Tobón Uribe } \\
\text { (HPTU). }\end{array}$ & Reintubação orotraqueal; Transporte para fora da UTI. \\
\hline $\begin{array}{l}\text { Risk Factors for Ventilator- Associates Events: } \\
\text { A Case-Control Multivariable Analysis. Estados } \\
\text { Unidos, } 2014 .\end{array}$ & $\begin{array}{l}\text { Identificar fatores de risco para condições associadas ao } \\
\text { ventilador e complicações relacionadas ao ventilador } \\
\text { relacionadas à infecção. }\end{array}$ & $\begin{array}{l}\text { Posicionamento incorreto da maca; Ausência de higiene oral; Risco de trombose } \\
\text { venosa profunda e úlcera; Exposição a sedação e analgesia. }\end{array}$ \\
\hline $\begin{array}{l}\text { Prognostic Risk Factors in Ventilator } \\
\text { Associated. Pneumonia. Turquia, } 2018\end{array}$ & $\begin{array}{l}\text { Identificar as características epidemiológicas e os fatores } \\
\text { que afetam o prognóstico dos pacientes em } \\
\text { acompanhamento ou tratamento para PAVM no } \\
\text { Departamento de Anestesiologia e Cuidados Críticos. }\end{array}$ & $\begin{array}{l}\text { Idade avançada; Presença de doença do sistema respiratório ou cardiovascular; } \\
\text { Falência de órgãos; Queimaduras; Trauma; Síndrome do desconforto respiratório } \\
\text { agudo; Colonização das vias respiratórias; Sinusite; Aspiração gástrica de alto } \\
\text { volume; Alterações sazonais. }\end{array}$ \\
\hline $\begin{array}{l}\text { Risk factors for the development of nosocomial } \\
\text { pneumonia and mortality on intensive care } \\
\text { units: application of competing risks models. } \\
\text { Alemanha, } 2008\end{array}$ & $\begin{array}{l}\text { Identificar possíveis fatores de risco para pneumonia } \\
\text { nosocomial em UTIs. }\end{array}$ & $\begin{array}{l}\text { Cirurgia eletiva ou cirurgia de emergência; Internação prévia; Uso de sonda } \\
\text { nasogástrica; VM. }\end{array}$ \\
\hline $\begin{array}{l}\text { Hyperoxemia as a risk factor for ventilator- } \\
\text { associated pneumonia. França, } 2016 \text {. }\end{array}$ & Determinar a relação entre hiperoxemia e PAVM. & Hiperóxia; Atelectasia; Depuração bacteriana reduzida. \\
\hline Incidência da PAVM em UTI. Brasil, 2017. & $\begin{array}{l}\text { Determinar a incidência de PAVM, bem como seus fatores } \\
\text { de risco, agentes etiológicos e evolução dos pacientes em } \\
\text { uma UTI de um hospital universitário no norte de Minas } \\
\text { Gerais. }\end{array}$ & $\begin{array}{l}\text { Tecnologia altamente especializada; Uso indiscriminado de antimicrobiano } \\
\text { prévio; Gravidade da doença de base; Tempo de permanência prévio em outras } \\
\text { unidades ou instituições de saúde; Tempo de permanência na UTI > 15 dias; } \\
\text { Procedimentos invasivos; Idade elevada; Maior escore de gravidade; DPOC; } \\
\text { Doença neurológica; Trauma; Cirurgia; Duração da VM; Reintubação; } \\
\text { Traqueostomia; CNG ou CNE; Dieta enteral; Aspiração de conteúdo gástrico; } \\
\text { Posição na maca. }\end{array}$ \\
\hline
\end{tabular}




\begin{tabular}{|l|}
\hline \multicolumn{1}{|c|}{ Título do Artigo, País e Ano de Publicação } \\
\hline $\begin{array}{l}\text { Infecção em pacientes sob ventilação artificial: } \\
\text { compreensão e medidas preventivas adotadas } \\
\text { por estudantes de enfermagem. Brasil, } 2013 .\end{array}$ \\
\hline $\begin{array}{l}\text { PAVM: discursos de profissionais acerca da } \\
\text { prevenção. Brasil, 2014. }\end{array}$ \\
\hline $\begin{array}{l}\text { PAVM: epidemiologia e impacto na evolução } \\
\text { clínica de pacientes em uma UTI. Brasil, 2009. }\end{array}$ \\
\hline
\end{tabular}

PAVM: fatores de risco. Brasil, 2011.

Prevalencia y factores de riesgo de la neumonía asociada com la asistencia respiratoria mecánica en una unidad de cuidados intensivos de adultos, Cúcuta, Colombia. Colômbia, 2017.

Prevalência e prognóstico dos pacientes com PAVM em um hospital universitário. Brasil, 2006.

Risk factors for device-associated infection related to organisational characteristics of intensive care units: findings from the Korean Nosocomial Infections Surveillance System. Coreia do Sul, 2010.

Treatment of pneumonia associated with mechanical and hospital ventilation: risk factors and impact on outcomes. Turquia, 2012.

Ventilator-associated pneumonia in an adult clinical-surgical intensive care unit of a Brazilian university hospital: incidence, risk factors, etiology, and antibiotic resistance. Brasil, 2008.

\section{Objetivo}

Investigar a compreensão de estudantes de enfermagem sobre a prevenção de infecção em pacientes sob ventilação artificial na UTI.

Identificar os cuidados que os profissionais consideram importantes para prevenção da PAVM.

Avaliar o impacto da PAVM na evolução clínica dos pacientes.

Descrever e analisar características epidemiológicas, clínicas, laboratoriais e fatores de risco em PAVM.

Prevalência e fatores de risco do uso de ventilação mecânica que implica o desenvolvimento de pneumonia na unidade de terapia intensiva.

Determinar prevalência de PAVM em UTI, fatores associados e evolução.

Avaliar os fatores de risco para DAls (Infecções associadas ao dispositivo) em UTIs.

Identificar os fatores de risco para terapia antimicrobiana inicial inadequado e determinar seu impacto subsequente nos resultados da PAVM e da pneumonia adquirida no hospital

Avaliar características clínicas, etiologia e resistência a agentes antimicrobianos e em pacientes com PAVM.

Legenda: PAVM: Pneumonia Associada à Ventilação Mecânica; UTI: Unidade de Terapia Intensiva. CNG: Cateter Nasogástrico; CNE: Cateter Nasoentérico. Fonte: Pinho MT, et al., 2021.

Higiene das mãos de profissionais de saúde; Procedimento invasivo; Perda da barreira protetora do paciente; Equipamentos de manuseio; Imunidade suprimida do paciente; Cuidado negligenciado dos profissionais; Ambiente; Tubo endotraqueal como reservatório de microrganismos; Aspirado como veículo de propagação de microorganismos.

Idade; Escore de gravidade; Doenças neurológicas; Traumas; Cirurgias.

Tempo de VM; Tempo de permanência na UTI e hospital; Uso de antibióticos; Fatores microbiológicos; Idade; Traqueostomia; Indicação da VM; Categoria diagnóstica.

Traqueostomia

Distúrbios respiratórios; Trauma; Edema pulmonar; Infecção urinária; Exposição prévia a antibióticos; Idade avançada.

Uso de VM; Intubação; Traqueostomia; Uso de vasopressores; Insuficiência respiratória; Pneumonia hospitalar e comunitária prévia a internação na unidade de terapia intensiva.

Unidade de terapia intensiva Cirúrgica

Tratamento com antibióticos prévio; Idade; Avaliação dos niveis de APACHE; Imudeficiência.

Tempo de ventilação mecânica maior que sete dias; Uso da VM; Traqueostomia; Uso de três antibióticos ou mais; Intubação endotraqueal; Tempo de internação na UTI; Exposição prévia a antibióticos; Idade > 60 anos; DPOC; Posicionamento no leito; Traumatismo craniano; Coma; Tratamento com esteroides; Tempo de permanência hospitalar; Cânulas endotraqueais; Cateteres intravasculares; Práticas inadequadas de controle de infecção. DPOC: Doença Pulmonar Obstrutiva Crônica; VM: Ventilação Mecânica; ; 


\section{DISCUSSÃO}

Esta revisão permitiu identificar os fatores de risco relacionados à PAVM em pacientes adultos internados em UTIs. Identificou-se que os principais fatores estão relacionados às condições clínicas, idade, uso de medicamentos, assistência em saúde e os dispositivos médicos. Dentre as condições clínicas, a presença de problemas respiratórios, no geral, torna o paciente mais vulnerável para o desenvolvimento de infeções do trato respiratório, como as pneumonias. Essas condições podem causar inflamação e obstrução das vias aéreas, impedindo a eliminação efetiva das secreções e propiciando a multiplicação de possíveis microrganismos presentes, que podem migrar para os pulmões (GUIMARÃES MMQ e ROCCO JR, 2006; KARAKUZU Z, et al., 2018).

A literatura destaca que a Doença Pulmonar Obstrutiva Crônica (DPOC), por compreender um grupo de doenças que levam a uma diminuição da taxa de fluxo de ar nos pulmões durante a expiração, prejudicam a respiração e causam lesões pulmonares irreversíveis Por ser um fator predisponente para o diagnóstico de atelectasia, a DPOC e sua respectiva gravidade clínica, aumenta a probabilidade do uso de ventilação mecânica invasiva e por consequência, uma possível colonização pulmonar (KARAKUZU Z, et al., 2018; GUIMARÃES MMQ e ROCCO JR, 2006).

Diante do exposto, é válido ressaltar que o sistema respiratório é dotado de defesas que auxiliam o organismo a evitar que patógenos se aloquem em seu interior. A exemplo disso, há o fluxo salivar que auxilia na limpeza das superfícies das vias aéreas, bem como o próprio mecanismo de tosse, que expulsa as partículas e os patógenos. Nos pacientes críticos, esses processos de defesa natural ficam comprometidos, elevando o risco de desenvolver pneumonia por aspiração (BATISTA JF, et al., 2013).

Além dos aspectos respiratórios, outras condições como a desnutrição, imunodeficiência, a gravidade das doenças de base, fatores microbiológicos, infecção do trato urinário, comorbidades cardíacas, comprometimento das vias aéreas, colonizações prévias, doenças neurológicas, choque e o risco de desenvolver trombose e úlcera são condições que podem ser observadas na clínica dos pacientes e que são fatores prejudiciais para os mecanismos de defesa do hospedeiro e, consequentemente, elevam a predisposição ao desenvolvimento da PAVM (MOTA EC, et al., 2017; SILVA SG, et al., 2014).

Outra situação encontrada na literatura, associa o diagnóstico de hemorragia digestiva, com o pH gástrico mais ácido $(<4)$, ao desenvolvimento de PAVM, de modo que a inibição da secreção gástrica com bloqueadores de receptores de histamina $(\mathrm{H} 2)$ ou da bomba de prótons e/ou dieta enteral favorecem a ocorrência da complicação estudada (GUIMARÃES MMQ e ROCCO JR, 2006).

Algumas condições inesperadas também se apresentam como fator de risco para a PAVM, como a ocorrência de traumas. Embora pacientes que sofreram traumas estejam mais propensos ao risco de desenvolvimento da pneumonia, a relação entre os agravos não foi diretamente elucidada pelos estudos (ROCHA LA, et al., 2008; SILVA SG, et al., 2014; MOTA, EC et al., 2017; KARAKUZU Z, et al., 2018). Dentre os mecanismos de trauma, o traumatismo cranioencefálico (TCE) grave se relaciona com o desenvolvimento da pneumonia, uma vez que gera lesões cerebrais e essas são causas frequentes de necessidade de VM prolongada, o que compromete a fisiologia do sistema respiratório e aumenta as complicações associadas à exposição dos riscos para a PAVM (FAEZ DCS, 2016).

A idade avançada, embora seja um fator intrínseco, também está relacionada com pior prognóstico. Na literatura esse fator é descrito como um risco não modificável para o desenvolvimento da PAVM. Nos estudos analisados, verificou-se que os pacientes maiores de 60 anos são mais susceptíveis ao desenvolvimento da pneumonia, assim como outras complicações clínicas. Essa relação pode ser explicada pelo declínio fisiológico e diminuição da resposta imunológica dos pacientes decorrentes do processo de envelhecimento. Além disso, os idosos, comumente, desenvolvem doenças crônicas que os deixam mais vulneráveis, aumentando o tempo de internação e a exposição aos riscos para o desenvolvimento da pneumonia nos hospitais (GUIMARÃES MMQ e ROCCO JR, 2006; RODRIGUES PMA, et al., 2009; MOTA EC, et al., 2016; SILVA SG, et al., 2014; KARAKUZU Z, et al., 2018).

Identificou-se também, que o uso prévio e inadequado de antibióticos (AB) é um fator independente para desenvolvimento da PAVM. Pacientes que fizeram uso recente e/ou indiscriminado da medicação em questão 
têm maior risco de apresentar resistência bacteriana (SELIGMAN R, et al., 2013; MOTA EC, et al., 2017). Além de antibioticoterapia prévia, outras medicações se associam com o maior risco de desenvolver PAVM, dentre eles destacam-se sedativos, analgésicos, vasopressores e esteroides (KARAKUZU Z, et al., 2018; GUIMARÃES MMQ e ROCCO JR, 2006; ROCHA LA, et al., 2008).

Dentre as medicações supracitadas, evidencia-se o uso de altas doses e/ou uso prolongado de sedativos. Essas medicações comprometem as funções fisiológicas do sistema respiratório do paciente, fazendo com que este fique dependente do uso do ventilador por maior tempo. Na tentativa de redução de riscos, é necessário adotar a prática de interrupção diária da sedação, quando possível. Essa estratégia permite observar, diariamente, a possibilidade de extubação do paciente crítico, buscando reduzir o tempo de exposição ao ventilador e seus riscos. Entretanto, é importante que a equipe prestadora da assistência avalie criteriosamente a decisão de extubar o paciente, uma vez que, quando realizada sem critérios, pode resultar na necessidade de reintubação e na adição de riscos (AMARAL JM e IVO OP, 2016).

Quando os pacientes intubados são submetidos à VM, os mecanismos de defesa do pulmão sofrem significativas alterações, modificando a fisiologia respiratória. A literatura aponta taxas de pneumonia que variam entre $9 \%$ e $67 \%$ entre todos os pacientes submetidos à ventilação mecânica, sendo esse o fator de risco mais relacionado com o aumento do índice de morbidade e mortalidade por infecções hospitalares (SILVA SG, et al., 2014; MOTA EC, et al., 2017).

Embora as discussões sobre o tempo de exposição ao ventilador e o aumento dos riscos seja controvérsia, a literatura aponta que o uso de ventilador mecânico por mais de sete dias é um fator de risco importante para 0 aumento da vulnerabilidade das vias aéreas do paciente. A literatura revela que uma estratégia preventiva para a PAVM é a redução do tempo de exposição à VM, de modo que as instituições implementem protocolos facilitadores do desmame precoce, além de priorizar, quando possível, o uso da ventilação mecânica não invasiva para evitar possíveis reintubações e maiores danos para o paciente exposto (ROCHA LA, et al., 2008).

Outros fatores de risco apontados pela literatura são os relacionados ao uso de dispositivos médicos como cateter nasogástrico/nasoentérico e a traqueostomia (ROCHA LA, et al., 2008). Os dispositivos estão diretamente relacionados ao aumento do risco de desenvolvimento de infecção, uma vez que são corpos estranhos que estão localizados próximos ou no próprio trato respiratório do paciente. Além disso, quanto mais grave clinicamente, mais dispositivos serão utilizados pelo paciente (SACHETTI A, et al., 2014; MOTA EC, et al., 2017; RODRIGUES PMA, et al.,2009; SILVA RM, et al., 2011 e GUIMARÃES MMQ e ROCCO JR, 2006).

Por mais que os pacientes internados em UTI se beneficiem de cuidados multiprofissionais intensivos e altas tecnologias, os mesmos acabam sendo submetidos a um alto número de procedimentos e uso de dispositivos invasivos, tornando-os mais vulneráveis às infecções e gerando a necessidade de atenção dos profissionais de saúde em relação aos possiveis eventos assistenciais (MOTA EC, et al., 2017). Diante disso, percebe-se a importância da utilização de dispositivos disponíveis que permitem a padronização do cuidado, abordando informações para a redução do desenvolvimento da complicação estudada, como por exemplo, o uso dos bundles (COELHO D, et al., 2020).

O bundle de ventilação se configura como um conjunto de medidas baseadas em evidências científicas que auxiliam os profissionais de saúde na identificação de possíveis riscos para o des 1envolvimento da PAVM e que, tais medidas associadas a assistência em saúde segura, contribuem para a prevenção de diversas complicações que os pacientes em uso de ventilação mecânica possam desenvolver, como por exemplo: sangramento gastrointestinal induzido por estresse, tromboembolismo venoso e, de fato, a pneumonia associada à ventilação mecânica (COELHO D, et al., 2020).

Nos cuidados diários ao paciente hospitalizado, a equipe de enfermagem desempenha um importante papel, atuando na assistência direta, buscando garantir a melhora do quadro clínico e prevenção de agravos. Assim sendo, é essencial que os profissionais realizem as ações baseadas nas medidas de prevenção da PAVM e de outras infecções relacionadas à assistência de saúde e ao risco do uso de dispositivos médicos (IRAS) (PAZ JS, et al., 2019). 
Os artigos analisados descrevem que, apesar de a higiene oral ser um cuidado básico e de baixo custo, muitas vezes é negligenciado pelo profissional. A não execução da correta higienização oral, embora muitas vezes subestimada, está diretamente relacionada ao desenvolvimento da PAVM. O paciente em uso da VM fica impossibilitado de mastigar e deglutir, favorecendo a hipossalivação e a formação do biofilme dental, que é considerado fator para o desenvolvimento de patógenos. Portanto, a ausência e/ou a inadequada realização da higiene oral e a presença de secreções espessas (constituídas de muco e microrganismos) favorecem a migração dos patógenos para a região subglótica e, assim, posteriormente para a árvore brônquica. $A$ migração leva ao acúmulo de muco nos pulmões, desencadeando o processo infeccioso. A higienização adequada deve ser realizada com a Clorexidina $0,12 \%$, na forma aquosa, uma vez que é um reconhecido antisséptico, com ação bactericida (SPEZZIA S, 2019).

Outro importante cuidado assistencial é a higienização das mãos do profissional. A baixa adesão a essa prática se torna um dado preocupante, pois essa é uma das principais medidas para a prevenção e controle da transmissão de infecções em geral. A higiene, quando realizada de maneira correta, reduz a carga microbiótica das mãos dos profissionais. Essa prática, quando associada ao correto uso de equipamento de proteção individual (EPI) contribui para prevenção da PAVM e sobretudo de outros processos infecciosos (SILVA AAL e GAZZINELI A, 2017; SILVA SG, et al., 2014; BATISTA JF, et al., 2013).

Além dos fatores já citados, o posicionamento do paciente no leito está diretamente correlacionado com a ocorrência da PAVM. Casualmente, pacientes permanecem posicionados incorretamente nos leitos. Além de causar desconforto e danos físicos, a posição no leito pode favorecer a translocação de bactérias do trato gastrointestinal (BATISTA JR, et al., 2013). Portanto, manter a cabeceira elevada entre 30 a 45으, se não houver contra-indicação, é um cuidado de enfermagem básico. Esse fator de risco se relaciona diretamente com os pacientes em uso de sonda nasoentérica, devido ao risco de broncoaspiração e, consequentemente, a ocorrência da pneumonia. Além disso, manter o paciente posicionado corretamente contribui para a melhoria do volume corrente e ventilatório, minimizando ocorrência de atelectasia (SILVA SG, et al., 2014).

A ventilação mecânica é, muitas vezes, indispensável para o tratamento de pacientes críticos, entretanto, é o fator de risco para o desenvolvimento da pneumonia associada à ventilação mecânica. A PAVM é considerada uma patologia de alta letalidade nas UTI's, devido sua complexidade. Diante disso, é importante a identificação dos fatores de risco relacionados a este problema, uma vez que esse conhecimento prévio possibilita que os profissionais, através de suas ações e intervenções, busquem reduzir o desenvolvimento da pneumonia. O trabalho tem como limitação a potencial heterogeneidade dos estudos selecionados e a necessidade de atualização constante.

\section{CONSIDERAÇÕES FINAIS}

A partir dessa revisão, pode-se observar diversos fatores de riscos relacionados à PAVM, dentre eles, a idade elevada, presença de doenças respiratórias ou outras doenças de base, fatores relacionados às práticas assistenciais, como a correta higiene das mãos, realização da higiene oral do paciente, adequado posicionamento no leito e manuseio de dispositivos médicos. É essencial que a equipe multiprofissional tenha conhecimento acerca da PAVM e das medidas que minimizem o agravo. Espera-se que a descrição dos fatores de risco obtidos através dessa revisão de literatura possa auxiliar na tomada de decisões e sensibilizar os profissionais de saúde acerca da importância de ações para prevenção da PAVM.

\section{REFERÊNCIAS}

1. ÁlvareZ LR, DUARTE AL. Prevalencia Y Factores De Riesgo De La Neumonía Asociada Con La Asistencia Respiratoria Mecánica En Una Unidad De Cuidados Intensivos De Adultos, Revista De Investigación En Salud. Universidad De Boyacá, Colômbia, Cucutá, 2017; 4(2): 248-266.

2. AMARAL JM, IVO OP. Prevenção de pneumonia associada à ventilação mecânica: um estudo observacional. Revista Enfermagem Contemporânea, 2016; 5(1): 109-117.

3. ANVISA. Medidas de Prevenção de Infecção Relacionada à Assistência à Saúde. 2017. Diponivel em: http://portal.anvisa.gov.br/documents/33852/3507912/Caderno+4++Medidas+de+Preven\%C3\%A7\%C3\%A3o+de+In fec\%C3\%A7\%C3\%A3o+Relacionada+\%C3\%A0+Assist\%C3\%AAncia+\%C3\%A0+Sa\%C3\%BAde/a3f23dfb-2c544e64-881c-fccf9220c373. Acessado em: 14 de junho de 2020. 
4. BATISTA JF, et al. Infecção em pacientes sob ventilação artificial: compreensão e medidas preventivas adotadas por estudantes de enfermagem. Journal of Nuersing UFPE on line, 2013; 7(4): 1120-1127.

5. BAYONA CEA, et al. Factores de riesgo para neumonia asociada al ventilador en un hospital de tercer nivel de la ciudad de Medellin: estudio de casos y controles. Medicina UPB, MEDICINA UPB Colómbia, 2008; 27(1): 25-32.

6. COELHO D, et al. O impacto da utilização de Bundles na prevenção da pneumonia associada a ventilação mecânica. REAID [Internet], 2020; 92(30).

7. COELHO-CASTELO AAM, et al. Resposta imune a doenças infecciosas. Medicina (Ribeirão Preto), Barretos, 2009; 42(2): 127-42.

8. DUTRA LA, et al. Pneumonia associada à ventilação mecânica: percepção dos profissionais de enfermagem. Rev enferm UFPE on line, Recife, 2019; 13(4): 884-92.

9. FAEZ DCS. Análise da retirada da ventilação mecânica dos pacientes vítimas de traumatismo craniencefálico grave. Tese de conclusão de Mestrado. Faculdade de Ciências Médicas da Universidade Estadual de Campinas, 2016.

10. GIARD M, et al. Early- and late-onset ventilator-associated pneumonia acquired in the intensive care unit: comparison of risk factors. Journal of Critical Care, França, 2008; 23(1): 27-33.

11. GUIMARÃES MMQ, ROCCO JR. Prevalência e prognóstico dos pacientes com pneumonia associada à ventilação mecânica em um hospital universitário. J. bras. Pneumol, São Paulo, 2006; 32(4).

12. KARAKUZU Z, et al. Risk Factors in Ventilator-Associated Pneumonia. Med Sci Monit, 2018; 5(24): $1321-1328$.

13. KWAK YG, et al. Prognostic Risk Factors for Device-Associated Infection Related to Organisational Characteristics of Intensive Care Units: Findings From the Korean Nosocomial Infections Surveillance System. J Hosp Infect, 2010; 75(3): 195-9.

14. LEAL GA, et al. Cuidados de Enfermagem para a Prevenção da Pneumonia Associada à Ventilação Mecânica em Unidades de Terapia Intensiva: Uma Revisão Literária. Cad Grad Cienc Biol Saúde [Internet], 2017; 4(1): 95-108.

15. LEWIS S, et al. Risk Factors for Ventilator-Associated Events: A Case-Control Multivariable Analysis. Crit Care Med, 2014; 42(8): 1839-1848.

16. MATOSO LML, CASTRO CHA. Indissociabilidade Clínica e Epidemiológica da Pneumonia. CATUSSABA. Revista cientifica da Escola e Saúde, 2013.

17. MENDES KDS, et al. Revisão integrativa: método de pesquisa para incorporação de evidências em saúde e enfermagem. Texto Contexto Enferm, 2008; 17(4): 756-764.

18. MOTA EC, et al. Incidência da pneumonia associada à ventilação mecânica em unidade de terapia intensiva. Medicina (Ribeirão Preto, Online.), 2017; 50(1): 39-46: 20.

19. NASCIMENTO TBP, et al. Efetividade das medidas de prevenção e controle de Pneumonia Associada à Ventilação Mecânica na UTI. Perspectivas Online. 2017; 7(17): 1-24.

20. PAZ S, et al. Medidas preventivas aplicadas à prevenção da pneumonia associada à ventilação mecânica na atuação em enfermagem- Revisão integrativa. Rev Inic Cient Ext, 2019; 2(2): 301-13.

21. PISKIN N, et al. Treatment of pneumonia associated with mechanical and hospital ventilation: risk factors and impact on outcomes BMC Infections Diseases 2012; 12(268): 2-9.

22. POMBO CMN, et al. Conhecimento dos profissionais de saúde na Unidade de Terapia Intensiva sobre prevenção de pneumonia associada à ventilação mecânica. Ciênc. Saúde coletiva, Rio de Janeiro, 2010; 15(1).

23. ROCHA LA, et al. Ventilator-associated pneumonia in an adult clinical-surgical intensive care unit of a Brazilian university hospital: incidence, risk factors, etiology, and antibiotic resistance. Braz J Infect Dis, Salvador, 2008; 12(1).

24. RODRIGUES PMA, et al. Pneumonia associada à ventilação mecânica: epidemiologia e impacto na evolução clínica de pacientes em uma unidade de terapia intensiva. J. bras. Pneumol, São Paulo, 2009; 35(11).

25. SACHETTI A, et al. Adesão às medidas de um bundle para prevenção de pneumonia associada à ventilação mecânica. Rev. bras. ter. intensiva, São Paulo, 2014; 26(4).

26. SANTOS CR, et al. Fatores de risco que favorecem a pneumonia associada à ventilação mecânica. Rev enferm UFPE on line., Recife, 2018; 12(12): 3401-15.

27. SELIGMAN R, et al. Fatores de risco para multirresistência bacteriana em pneumonias adquiridas no hospital não associadas à ventilação mecânica. Jornal Brasileiro de Pneumologia, 2013; 39(3).

28. SILVA AAL, GAZZINELI A. Knowledge of nursing professionals in relation to measures of prevention of infections. Rev Enferm UFPE on line, 2017; 11(1): 18-23.

29. SILVA RM, et al. Pneumonia associada à ventilação mecânica: fatores de risco. Rev Bras Clin Med, São Paulo, 2011; 9(1): 5-10.

30. SILVA SG, et al. Pneumonia associada à ventilação mecânica: discursos de profissionais acerca da prevenção. Esc. Anna Nery, 2014;18(2).

31. SILVA SG, et al. Pneumonia associada à ventilação mecânica: discursos de profissionais acerca da prevenção. Esc. Anna Nery, Rio de Janeiro, 2014; 18(2).

32. SIX S, et al. Hyperoxemia as a Risk Factor for Ventilator-Associated Pneumonia. Crit Care, 2016; 22: 20 (1): 195.

33. SPEZZIA S. Pneumonia nosocomial, biofilme dentário e doenças periodontais. Braz J Periodontol, 2019; 29(2): 6572.

34. WOLKEWITZ. M, et al. Risk factors for the development of nosocomial pneumonia and mortality on care units: application of competing risks models. Crit Care, 2008; 12(2). 